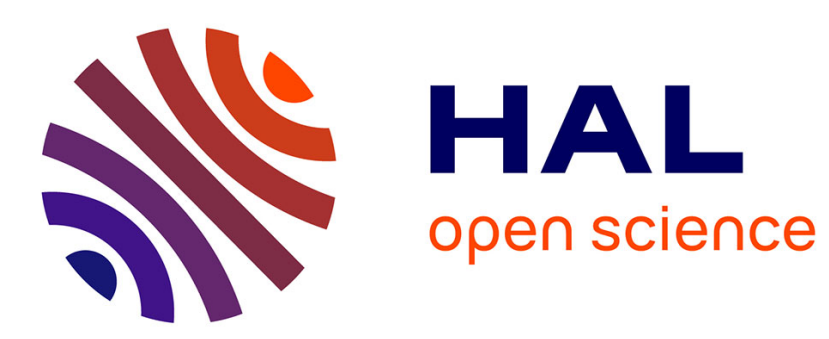

\title{
The Electromagnetic Interior Transmission Problem for Regions with Cavities
}

\author{
Anne Cossonnière, Houssem Haddar
}

\section{To cite this version:}

Anne Cossonnière, Houssem Haddar. The Electromagnetic Interior Transmission Problem for Regions with Cavities. SIAM Journal on Mathematical Analysis, 2011, 43 (4), pp.1698-1715. 10.1137/100813890 . hal-00743733

\section{HAL Id: hal-00743733 \\ https://hal.inria.fr/hal-00743733}

Submitted on 19 Oct 2012

HAL is a multi-disciplinary open access archive for the deposit and dissemination of scientific research documents, whether they are published or not. The documents may come from teaching and research institutions in France or abroad, or from public or private research centers.
L'archive ouverte pluridisciplinaire HAL, est destinée au dépôt et à la diffusion de documents scientifiques de niveau recherche, publiés ou non, émanant des établissements d'enseignement et de recherche français ou étrangers, des laboratoires publics ou privés. 


\title{
THE ELECTROMAGNETIC INTERIOR TRANSMISSION PROBLEM FOR REGIONS WITH CAVITIES
}

\author{
ANNE COSSONNIÈRE* AND HOUSSEM HADDAR ${ }^{\dagger}$
}

\begin{abstract}
We consider the electromagnetic interior transmission problem in the case when the medium has cavities, i.e. regions in which the index of refraction is the same as in the host medium. We address the configuration where the electromagnetic permeability is constant while the electric permittivity is variable and may be anisotropic. In this case, using appropriate reformulation of the problem into a fourth order pde, we establish the Fredholm property for this problem and show that transmission eigenvalues exist and form a discrete set. Monotonicity properties of the first eigenvalue in terms of the permittivity and the size of the cavity are established.
\end{abstract}

Key words. Interior transmission problem, inhomogeneous medium, transmission eigenvalues.

AMS subject classifications. 35R30, 35Q60, 35J40, 78A25.

Introduction. The interior transmission problem (ITP) is by now a well known problem in inverse scattering theory that plays a fundamental role in the theoretical and numerical investigations of the inverse medium problem $[7,11,1,10,12]$. A particular attention has been given in recent years to the study of the frequencies for which this problem has non unique solutions : the so-called transmission eigenvalues. These values can for instance be used to obtain bounds of the inclusion physical parameters $[4,2,8]$, or more importantly in non destructive testing of complex materials [3]. The latter application motivates the present work, where ITP is considered for anisotropic inclusions that may contain some cavities: i.e. regions where the index of refraction has the same value as the exterior medium. Mathematically, the cavity region corresponds with a degenerate form of the ITP (the two fields satisfy the same equation) and therefore causes difficulties in extending the techniques used for "regular forms". A first study of this configuration was initiated in [3] for the scalar problem. We provide here an extension of this work to the full electromagnetic problem by following a similar route : the ITP is reformulated as a fourth order pde outside the cavity region and the latter is taken into account as a constrain in the variational space. Besides the technicality inherent to Maxwell's equations, the main difficulty here is in proving the equivalence between weak and variational solutions and is in finding the appropriate splitting of the variational form into coercive and compact parts. In a second step, and inspired by the recent works [6, 5], we use this formulation to prove the existence of an infinite discrete set of transmission eigenvalues for general cases and prove a monotonicity property with respect to the cavity size of the first eigenvalue. The main difficulty in this part lies in the fact that the variational space depends of the frequency. The introduction of a projection operator and continuity properties of the latter with respect to the frequency are used in order to solve the problem.

The paper is organized as follows. The first section is dedicated to introducing the ITP and establishing the appropriate variational formulation of the problem. The Fredholm property of the obtained problem is then proved. The second section is devoted to the study of transmission eigenvalues : we prove the existence of an infinite discrete set of these special frequencies in the case without absorption and establish

${ }^{*}$ CERFACS, 42 avenue Gaspard Coriolis 31057 Toulouse Cedex 01, France (cosso@cerfacs.fr)

${ }^{\dagger}$ INRIA Saclay Ile de France/CMAP Ecole Polytechnique, Route de Saclay, 91128 Palaiseau Cedex, France (haddar@cmapx.polytechnique.fr) 
monotonicity properties with respect to the cavity size and the medium index. Finally, the appendix gives a useful result on $L^{2}$ solutions to Maxwell's equations in a given domain.

1. Interior transmission problem. Let $D \subset \mathbb{R}^{3}$ be a simply connected and bounded region with a piece-wise smooth boundary $\partial D$. We denote by $(\cdot, \cdot)_{D}$ the $L^{2}(D)^{3}$ scalar product and consider the Hilbert spaces :

$$
\begin{gathered}
H(\operatorname{curl}, D):=\left\{\mathbf{u} \in L^{2}(D)^{3} / \operatorname{curl} \mathbf{u} \in L^{2}(D)^{3}\right\} \\
H_{0}(\operatorname{curl}, D):=\{\mathbf{u} \in H(\operatorname{curl}, D) / \nu \times \mathbf{u}=0 \text { on } \partial D\}
\end{gathered}
$$

equipped with the scalar product $(\mathbf{u}, \mathbf{v})_{\text {curl }}=(\mathbf{u}, \mathbf{v})_{D}+(\operatorname{curl} \mathbf{u}, \operatorname{curl} \mathbf{v})_{D}$ and the corresponding norm $\|\cdot\|_{\text {curl }}$. We also define

$$
\begin{gathered}
\mathcal{U}(D):=\{\mathbf{u} \in H(\operatorname{curl}, D) / \operatorname{curl} \mathbf{u} \in H(\operatorname{curl}, D)\} \\
\mathcal{U}_{0}(D):=\left\{\mathbf{u} \in H_{0}(\operatorname{curl}, D) / \operatorname{curl} \mathbf{u} \in H_{0}(\operatorname{curl}, D)\right\}
\end{gathered}
$$

equipped with the scalar product $(\mathbf{u}, \mathbf{v})_{\mathcal{U}}=(\mathbf{u}, \mathbf{v})_{\text {curl }}+(\operatorname{curl} \mathbf{u}, \operatorname{curl} \mathbf{v})_{\text {curl }}$ and the corresponding norm $\|\cdot\|_{\mathcal{U}}$.

We assume that $D$ contains a region $D_{0} \subset D$ which can possibly be multiply connected and with a piece-wise smooth boundary $\partial D_{0}$ and such that $D \backslash D_{0}$ is connected (see Fig. 1.1). Let $\nu$ denote the unit outward normal to $\partial D$ and $\partial D_{0}$. Let $N$ be a $3 \times 3$ symmetric matrix whose entries are bounded complex-valued functions in $\mathbb{R}^{3}$ and such that $N=I$ in $D_{0}$. This matrix will represent the medium index inside $D$ and We shall also assume that there exists a constant $\gamma>0$ such that either

$$
\operatorname{Re}\left((N-I)^{-1} \xi, \xi\right) \geq \gamma|\xi|^{2} \quad \text { or } \quad \operatorname{Re}\left((I-N)^{-1} \xi, \xi\right) \geq \gamma|\xi|^{2}
$$

for all $\xi$ in $\mathbb{C}^{3}$ and almost everywhere in $D \backslash \bar{D}_{0}$.

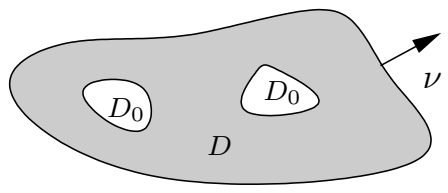

FIG. 1.1. Geometry and notation

Let $s \geq 0$ be a given real number and $H^{s}(\partial D)$ be the usual Sobolev space. We define

$$
T H^{s}(\partial D):=\left\{\varphi \in H^{s}(\partial D)^{3} / \varphi \cdot \nu=0\right\} .
$$

We consider the following interior transmission problem :

$$
\begin{gathered}
\text { curlcurl } \mathbf{E}-k^{2} N \mathbf{E}=0 \text { in } D \\
\text { curlcurl } \mathbf{E}_{0}-k^{2} \mathbf{E}_{0}=0 \text { in } D \\
\nu \times \mathbf{E}-\nu \times \mathbf{E}_{0}=\mathbf{G} \text { on } \partial D \\
\nu \times \operatorname{curl} \mathbf{E}-\nu \times \operatorname{curl} \mathbf{E}_{0}=\mathbf{H} \text { on } \partial D
\end{gathered}
$$


with $\mathbf{G}$ and $\mathbf{H}$ some given boundary data. We shall assume that there exists $\boldsymbol{\Theta} \in$ $H^{2}(D)^{3}$ such that

$$
\nu \times \mathbf{\Theta}=\mathbf{G} \text { and } \quad \nu \times \operatorname{curl} \boldsymbol{\Theta}=\mathbf{H} \text { on } \partial D,
$$

(see Remark 1.1 below). Using a cutoff function one can also guarantee that $\Theta=0$ in $D_{\Theta}$ where $D_{0} \subset D_{\Theta} \subset D$, which will be assumed in the sequel.

REMARK 1.1. It is proven in [10] that if $\partial D$ is sufficiently regular (e.g. $C^{3}$ ), then for any boundary data $\mathbf{G} \in T H^{3 / 2}(\partial D)$ and $\mathbf{H} \in T H^{1 / 2}(\partial D)$, there exists $\Theta \in H^{2}(D)^{3}$ such that (1.5) holds and such that

$$
\|\mathbf{\Theta}\|_{H^{2}(D)} \leq c\left(\|\mathbf{G}\|_{H^{3 / 2}}+\|\mathbf{H}\|_{H^{1 / 2}}\right)
$$

where $c$ is a constant independent of $\mathbf{G}$ and $\mathbf{H}$.

We define a weak solution to (1.1)-(1.4) as the pair of functions $\mathbf{E}$ and $\mathbf{E}_{0}$ in $L^{2}(D)$ solution to (1.1)-(1.2) in the distributional sense such that $\mathbf{F}=\mathbf{E}-\mathbf{E}_{0} \in \mathcal{U}(D)$ satisfies (1.3)-(1.4). Now, we consider two weak solutions $\mathbf{E}$ and $\mathbf{E}_{0}$ to (1.1)-(1.4). Then $\mathbf{F}=\mathbf{E}-\mathbf{E}_{0}$ satisfies :

$$
\operatorname{curl} \operatorname{curl} \mathbf{F}-k^{2} N \mathbf{F}=k^{2}(N-I) \mathbf{E}_{0} \quad \text { in } D \backslash \bar{D}_{0}
$$

or

$$
\text { curl curl } \mathbf{F}-k^{2} \mathbf{F}=k^{2}(N-I) \mathbf{E} \quad \text { in } D \backslash \bar{D}_{0}
$$

and we obtain :

$$
\left(\text { curlcurl }-k^{2} N\right)(N-I)^{-1}\left(\operatorname{curl} \operatorname{curl}-k^{2}\right) \mathbf{F}=0 \quad \text { in } D \backslash \bar{D}_{0}
$$

together with

$$
\nu \times \mathbf{F}=\mathbf{G} \quad ; \quad \nu \times \operatorname{curl} \mathbf{F}=\mathbf{H} \quad \text { on } \partial D .
$$

Moreover, inside $D_{0}, \mathbf{F}$ satisfies :

$$
\left(\text { curl curl }-k^{2}\right) \mathbf{F}=0
$$

with the continuity of the Cauchy data across $\partial D_{0}$ :

$$
\nu \times \mathbf{F}^{-}=\nu \times \mathbf{F}^{+} \quad ; \quad \nu \times \operatorname{curl} \mathbf{F}^{-}=\nu \times \operatorname{curl} \mathbf{F}^{+}
$$

where for a regular function $\mathbf{F}, \mathbf{F}^{ \pm}(x):=\lim _{h \rightarrow 0^{+}} \mathbf{F}\left(x \pm h \nu_{x}\right)$ for $x \in \partial D_{0}$ and $\nu_{x}$ the outward unit normal to $\partial D_{0}$.

However the latter equations are not sufficient to define $\mathbf{E}$ and $\mathbf{E}_{0}$ inside $\partial D_{0}$ so one needs to add an additional unknown inside $D_{0}$, for instance the function $\mathbf{E}$ that satisfies :

$$
\left(\text { curl curl }-k^{2}\right) \mathbf{E}=0 \text { in } D_{0}
$$


From the continuity of the Cauchy data of (1.6) we also get two more conditions on $\partial D_{0}$ :

$$
\begin{aligned}
\nu \times\left(\frac{1}{k^{2}}(N-I)^{-1}\left(\operatorname{curl} \operatorname{curl}-k^{2}\right) \mathbf{F}\right)^{+} & =\nu \times \mathbf{E}^{-} \\
\nu \times \operatorname{curl}\left(\frac{1}{k^{2}}(N-I)^{-1}\left(\operatorname{curl} \operatorname{curl}-k^{2}\right) \mathbf{F}\right)^{+} & =\nu \times \operatorname{curl} \mathbf{E}^{-}
\end{aligned}
$$

One can now easily verifies the following theorem :

Theorem 1.1. $\mathbf{F} \in \mathcal{U}(D)$ and $\mathbf{E} \in L^{2}\left(D_{0}\right)^{3}$ are solutions to (1.7)-(1.13) if and only if $\mathbf{E}$ and $\mathbf{E}_{0}$ are weak solutions to (1.1)-(1.4) with

$$
\mathbf{E}:=\frac{1}{k^{2}}(N-I)^{-1}\left(\operatorname{curl} \operatorname{curl}-k^{2}\right) \mathbf{F} \text { in } D \backslash \bar{D}_{0} \text { and } \mathbf{E}_{0}:=\mathbf{E}-\mathbf{F} .
$$

In order to study the existence and uniqueness of the solution, we will use a variational approach. The main difficulty is to define an appropriate variational space which will guarantee the well-posedness and the equivalence between the variational formulation and the system (1.7)-(1.13).

Let us define the Hilbert space

$$
V\left(D, D_{0}, k\right):=\left\{\mathbf{E} \in \mathcal{U}(D) / \operatorname{curl} \operatorname{curl} \mathbf{E}-k^{2} \mathbf{E}=0 \text { in } D_{0}\right\}
$$

equipped with the scalar product $(\cdot, \cdot)_{\mathcal{U}}$ and the closed subspace

$$
V_{0}\left(D, D_{0}, k\right):=\left\{\mathbf{E} \in \mathcal{U}_{0}(D) / \text { curl curl } \mathbf{E}-k^{2} \mathbf{E}=0 \text { in } D_{0}\right\} .
$$

Let $\mathbf{F} \in V\left(D, D_{0}, k\right)$ and consider a test function $\boldsymbol{\Psi} \in V_{0}\left(D, D_{0}, k\right)$. We assume that $\mathbf{F}$ and $\boldsymbol{\Psi}$ are regular enough to justify the various integrating by parts. Multiplying (1.7) by $\overline{\boldsymbol{\Psi}}$ and integrating by parts we obtain :

$$
\begin{aligned}
0= & \int_{D \backslash \bar{D}_{0}}\left(\operatorname{curl} \operatorname{curl}-k^{2} N\right)(N-I)^{-1}\left(\operatorname{curl} \operatorname{curl}-k^{2}\right) \mathbf{F} \cdot \overline{\mathbf{\Psi}} d x \\
= & \int_{D \backslash \bar{D}_{0}}\left(\left(\operatorname{curl} \operatorname{curl}-k^{2}\right)(N-I)^{-1}\left(\operatorname{curl} \operatorname{curl}-k^{2}\right) \mathbf{F}-k^{2}\left(\operatorname{curl} \operatorname{curl}-k^{2}\right) \mathbf{F}\right) \cdot \overline{\mathbf{\Psi}} d x \\
= & \int_{D \backslash \bar{D}_{0}}(N-I)^{-1}\left(\operatorname{curl} \operatorname{curl}-k^{2}\right) \mathbf{F} \cdot\left(\operatorname{curl} \operatorname{curl} \overline{\mathbf{\Psi}}-k^{2} \overline{\mathbf{\Psi}}\right) d x \\
& -k^{2} \int_{D \backslash \bar{D}_{0}}\left(\operatorname{curl} \operatorname{curl} \mathbf{F}-k^{2} \mathbf{F}\right) \cdot \overline{\mathbf{\Psi}} d x \\
& +\int_{\partial D_{0}} \nu \times\left((N-I)^{-1}\left(\operatorname{curl} \operatorname{curl} \mathbf{F}-k^{2} \mathbf{F}\right)\right) \cdot \operatorname{curl} \overline{\mathbf{\Psi}} d s \\
& -\int_{\partial D_{0}} \nu \times \overline{\mathbf{\Psi}} \cdot \operatorname{curl}\left((N-I)^{-1}\left(\operatorname{curl} \operatorname{curl} \mathbf{F}-k^{2} \mathbf{F}\right)\right) d s
\end{aligned}
$$

Using the fact that $\overline{\mathbf{\Psi}} \in V_{0}\left(D, D_{0}, k\right)$, the boundary conditions (1.12) and (1.13) and equation (1.11) imply that 


$$
\begin{aligned}
\int_{\partial D_{0}} \nu \times\left((N-I)^{-1}\right. & \left.\left(\operatorname{curl} \operatorname{curl} \mathbf{F}-k^{2} \mathbf{F}\right)\right) \cdot \operatorname{curl} \overline{\mathbf{\Psi}} d s \\
& -\int_{\partial D_{0}} \nu \times \overline{\mathbf{\Psi}} \cdot \operatorname{curl}\left((N-I)^{-1}\left(\operatorname{curl} \operatorname{curl} \mathbf{F}-k^{2} \mathbf{F}\right)\right) d s=0
\end{aligned}
$$

Therefore we finally have that

$$
\begin{aligned}
\int_{D \backslash \bar{D}_{0}}(N-I)^{-1}\left(\operatorname{curl} \operatorname{curl} \mathbf{F}-k^{2} \mathbf{F}\right) & \cdot\left(\operatorname{curl} \operatorname{curl} \overline{\mathbf{\Psi}}-k^{2} \overline{\mathbf{\Psi}}\right) d x \\
& -k^{2} \int_{D \backslash \bar{D}_{0}}\left(\operatorname{curl} \operatorname{curl} \mathbf{F}-k^{2} \mathbf{F}\right) \cdot \overline{\mathbf{\Psi}} d x=0
\end{aligned}
$$

which is required to be valid for all $\boldsymbol{\Psi} \in V_{0}\left(D, D_{0}, k\right)$.

Consequently the variational formulation amounts to finding $\mathbf{F}_{0}=\mathbf{F}-\boldsymbol{\Theta} \in$ $V_{0}\left(D, D_{0}, k\right)$ such that :

$$
\begin{aligned}
& \int_{D \backslash \bar{D}_{0}}(N-I)^{-1}\left(\operatorname{curl} \operatorname{curl} \mathbf{F}_{0}-k^{2} \mathbf{F}_{0}\right) \cdot\left(\operatorname{curl} \operatorname{curl} \overline{\mathbf{\Psi}}-k^{2} \overline{\mathbf{\Psi}}\right) d x \\
&-k^{2} \int_{D \backslash \bar{D}_{0}}\left(\operatorname{curl} \operatorname{curl} \mathbf{F}_{0}-k^{2} \mathbf{F}_{0}\right) \cdot \overline{\mathbf{\Psi}} d x \\
&=-\int_{D \backslash \bar{D}_{0}}(N-I)^{-1}\left(\operatorname{curl} \operatorname{curl} \boldsymbol{\Theta}-k^{2} \boldsymbol{\Theta}\right) \cdot\left(\operatorname{curl} \operatorname{curl} \overline{\mathbf{\Psi}}-k^{2} \overline{\mathbf{\Psi}}\right) d x \\
&+k^{2} \int_{D \backslash \bar{D}_{0}}\left(\operatorname{curl} \operatorname{curl} \mathbf{\Theta}-k^{2} \mathbf{\Theta}\right) \cdot \overline{\mathbf{\Psi}} d x
\end{aligned}
$$

for all $\boldsymbol{\Psi} \in V_{0}\left(D, D_{0}, k\right)$. In the following, this variational formulation will be used in the case where $(N-I)^{-1}$ is bounded positive definite. In the other case where $(I-$ $N)^{-1}$ is bounded positive definite, we shall use the equivalent variational formulation :

$$
\begin{array}{r}
\int_{D \backslash \bar{D}_{0}} N(I-N)^{-1}\left(\operatorname{curl} \operatorname{curl} \mathbf{F}_{0}-k^{2} \mathbf{F}_{0}\right) \cdot\left(\operatorname{curl} \operatorname{curl} \overline{\mathbf{\Psi}}-k^{2} \overline{\mathbf{\Psi}}\right) d x \\
+\int_{D \backslash \bar{D}_{0}}\left(\operatorname{curl} \operatorname{curl} \mathbf{F}_{0}-k^{2} \mathbf{F}_{0}\right) \cdot \operatorname{curl} \operatorname{curl} \overline{\mathbf{\Psi}} d x \\
=-\int_{D \backslash \bar{D}_{0}} N(I-N)^{-1}\left(\operatorname{curl} \operatorname{curl} \boldsymbol{\Theta}-k^{2} \boldsymbol{\Theta}\right) \cdot\left(\operatorname{curl} \operatorname{curl} \overline{\mathbf{\Psi}}-k^{2} \overline{\mathbf{\Psi}}\right) d x \\
-\int_{D \backslash \bar{D}_{0}}\left(\operatorname{curl} \operatorname{curl} \boldsymbol{\Theta}-k^{2} \mathbf{\Theta}\right) \cdot \operatorname{curl} \operatorname{curl} \overline{\mathbf{\Psi}} d x
\end{array}
$$

One can remark that the above variational formulations (1.16) and (1.17) involve only $\mathbf{F}$. The following lemma shows that the existence of $\mathbf{E}$ is implicitly contained in the variational formulation.

Definition 1.1. A real $\lambda$ is a called an eigenvalue for curlcurl in $D_{0}$ if there exists $\mathbf{v} \in H\left(\right.$ curl,$\left.D_{0}\right)$ a non trivial solution to

$$
\begin{cases}\operatorname{curl} \operatorname{curl} \mathbf{v}-\lambda \mathbf{v}=0 & \text { in } D_{0} \\ \mathbf{v} \times \nu=0 & \text { on } \partial D_{0} .\end{cases}
$$


REMARK 1.2. We remark that if $\lambda \neq 0$, then existence of a non trivial solution $\mathbf{v} \in H\left(\operatorname{curl}, D_{0}\right)$ to (1.18) is equivalent to existence of non trivial solution $\mathbf{v} \in H\left(\operatorname{curl}, D_{0}\right)$ to

$$
\begin{cases}\text { curl curl } \mathbf{v}-\lambda \mathbf{v}=0 & \text { in } D_{0} \\ \operatorname{curl} \mathbf{v} \times \nu=0 & \text { on } \partial D_{0} .\end{cases}
$$

LEMMA 1.2. We assume that $\partial D_{0}$ is a $C^{2}$ boundary and that $k^{2}>0$ is not an eigenvalue for curlcurl in $D_{0}$. Let $(\alpha, \beta) \in T H^{-3 / 2}\left(\partial D_{0}\right) \times T H^{-1 / 2}\left(\partial D_{0}\right)$ such that for all $\boldsymbol{\Psi} \in V_{0}\left(D, D_{0}, k\right) \cap H^{2}(D)$,

$$
\langle\nu \times \operatorname{curl} \boldsymbol{\Psi}, \beta\rangle_{T H^{1 / 2}, T H^{-1 / 2}}-\langle\alpha, \nu \times \boldsymbol{\Psi}\rangle_{T H^{-3 / 2}, T H^{3 / 2}}=0 .
$$

Then there exists $\mathbf{E} \in L^{2}\left(D_{0}\right)$ such that curlcurl $\mathbf{E}-k^{2} \mathbf{E}=0$ in $D_{0}$ and $(\nu \times \mathbf{E}, \nu \times$ $\operatorname{curl} \mathbf{E})=(\nu \times \beta, \nu \times \alpha)$ in $\partial D_{0}$.

Proof. Let $\mathbf{E} \in L^{2}\left(D_{0}\right)^{3}$ be a weak solution of curl curl $\mathbf{E}-k^{2} \mathbf{E}=0$ in $D_{0}$ and $\nu \times \mathbf{E}=\nu \times \beta$ on $\partial D_{0}$ (see Appendix theorem 3.1). Let $\boldsymbol{\Psi} \in V_{0}\left(D, D_{0}, k\right)$. Integrating by parts we obtain

$$
\begin{aligned}
\int_{D_{0}}\left(\operatorname{curl} \operatorname{curl} \mathbf{E}-k^{2} \mathbf{E}\right) \cdot \overline{\mathbf{\Psi}} d x= & \int_{D_{0}}\left(\operatorname{curl} \operatorname{curl} \overline{\mathbf{\Psi}}-k^{2} \overline{\boldsymbol{\Psi}}\right) \cdot \mathbf{E} d x \\
& +\int_{\partial D_{0}} \nu \times \mathbf{E} \cdot \operatorname{curl} \overline{\mathbf{\Psi}} d s-\int_{\partial D_{0}} \nu \times \overline{\mathbf{\Psi}} \cdot \operatorname{curl} \mathbf{E} d s
\end{aligned}
$$

Then

$$
\int_{\partial D_{0}} \nu \times \mathbf{E} \cdot \operatorname{curl} \overline{\mathbf{\Psi}} d s-\int_{\partial D_{0}} \nu \times \overline{\mathbf{\Psi}} \cdot \operatorname{curl} \mathbf{E} d s=0
$$

i.e.

$$
\int_{\partial D_{0}}(\alpha-\operatorname{curl} \mathbf{E}) \cdot \nu \times \overline{\mathbf{\Psi}} d s=0
$$

for all $\boldsymbol{\Psi} \in V_{0}\left(D, D_{0}, k\right) \cap H^{2}(D)$. Using the density theorem 3 in $[13]$ since $V_{0}\left(D, D_{0}, k\right)$ contains the set of Maxwell-Herglotz-fields, we can conclude that the traces of functions in $V_{0}\left(D, D_{0}, k\right)$ are dense in $T H^{3 / 2}\left(\partial D_{0}\right)$. Hence curl $\mathbf{E}=\alpha$. Then $\alpha \times \nu=$ $\operatorname{curl} \mathbf{E} \times \nu$ in $T H^{3 / 2}\left(\partial D_{0}\right)$.

We now can state the equivalence between weak solutions to (1.1)-(1.4) and solutions to the variational formulations (1.16) and (1.17).

THEOREM 1.3. Assume that $\partial D_{0}$ is a $C^{2}$ boundary and that $k^{2}>0$ is not an eigenvalue for curlcurl in $D_{0}$. Then the existence and uniqueness of a weak solution $\mathbf{E}$ and $\mathbf{E}_{0}$ to the interior transmission problem (1.1)-(1.4) is equivalent to the existence and uniqueness of a solution $\mathbf{F}_{0}$ to the variational problem (1.16) or (1.17).

Proof. It remains only to verify that a solution of (1.16) or (1.17) defines a weak solution $w$ and $v$ to the interior transmission problem (1.1)-(1.4). Let $\boldsymbol{\Psi}$ be a $\mathcal{C}^{\infty}$ function with compact support in $D \backslash \bar{D}_{0}$. From (1.15), we can show that $\mathbf{F}$ satisfies (1.8). In particular the function

$$
\mathbf{E}^{+}:=\left(-\frac{1}{k^{2}}(N-I)^{-1}\left(\operatorname{curl} \operatorname{curl} \mathbf{F}-k^{2} \mathbf{F}\right)\right)_{\mid D \backslash \bar{D}_{0}}
$$


satisfies $\mathbf{E}^{+} \in L^{2}\left(D \backslash \bar{D}_{0}\right)$ and curl curl $\mathbf{E}^{+}-k^{2} N \mathbf{E}^{+}=0$ in $D \backslash \bar{D}_{0}$. For an arbitrary test function $\boldsymbol{\Psi} \in V_{0}\left(D, D_{0}, k\right) \cap H^{2}(D)$, integrating by parts (1.15) we obtain

$$
\left\langle\nu \times \operatorname{curl} \boldsymbol{\Psi}, \mathbf{E}^{+}\right\rangle_{T H^{1 / 2}, T H^{-1 / 2}}-\left\langle\operatorname{curl} \mathbf{E}^{+}, \nu \times \mathbf{\Psi}\right\rangle_{T H^{-3 / 2}, T H^{3 / 2}}=0 .
$$

Applying lemma 1.2 we now obtain the existence of $\mathbf{E}^{-} \in L^{2}\left(D_{0}\right)$ satisfying (1.11)(1.13).

We now proceed with the proof of existence and uniqueness of a variational solution. In the following we exclude the values of $k$ for which the uniqueness does not hold, namely the so-called transmission eigenvalues.

DEFINITION 1.2. Values of $k>0$ for which the homogeneous variational problem (i.e. for $\boldsymbol{\Theta}=0$ ) has nontrivial solutions $\mathbf{F}_{0}$ are called transmission eigenvalues.

The following theorem concludes this section by proving the existence and uniqueness of a solution of the (ITP) using the Fredholm alternative.

Theorem 1.4. Assume that $N \in L^{\infty}\left(D, \mathbb{R}^{3 \times 3}\right)$. Then the interior transmission problem has a unique solution provided that $k$ is not a transmission eigenvalue. This solution depends continuously on the data $\boldsymbol{\Theta}$.

Proof. We first assume that $(N-I)^{-1}$ is bounded positive definite. Let us define the following bounded sesquilinear forms on $V_{0}\left(D, D_{0}, k\right) \times V_{0}\left(D, D_{0}, k\right)$ :

$\mathcal{A}_{k}(\mathbf{u}, \mathbf{v})=\int_{D \backslash \bar{D}_{0}}(N-I)^{-1}\left(\operatorname{curl} \operatorname{curl} \mathbf{u}-k^{2} \mathbf{u}\right) \cdot\left(\operatorname{curl} \operatorname{curl} \overline{\mathbf{v}}-k^{2} \overline{\mathbf{v}}\right) d x+k^{4} \int_{D} \mathbf{u} \cdot \overline{\mathbf{v}} d x$

and

$$
\begin{aligned}
\mathcal{B}_{k}(\mathbf{u}, \mathbf{v}) & =-k^{2} \int_{D \backslash \bar{D}_{0}} \overline{\mathbf{v}} \cdot \operatorname{curl} \operatorname{curl} \mathbf{u} d x-k^{4} \int_{D_{0}} \mathbf{u} \cdot \overline{\mathbf{v}} d x \\
& =-k^{2} \int_{D} \operatorname{curl} \mathbf{u} \cdot \operatorname{curl} \overline{\mathbf{v}} d x .
\end{aligned}
$$

The variational equation now becomes :

$$
\mathcal{A}_{k}\left(\mathbf{F}_{0}, \boldsymbol{\Psi}\right)+\mathcal{B}_{k}\left(\mathbf{F}_{0}, \boldsymbol{\Psi}\right)=-\mathcal{A}_{k}(\boldsymbol{\Theta}, \boldsymbol{\Psi})-\mathcal{B}_{k}(\boldsymbol{\Theta}, \boldsymbol{\Psi})
$$

Since $(N-I)^{-1}$ is bounded positive definite, there exists a constant $\gamma$ such that

$$
\mathcal{A}_{k}(\mathbf{u}, \mathbf{u}) \geq \gamma\left\|\operatorname{curl} \operatorname{curl} \mathbf{u}-k^{2} \mathbf{u}\right\|_{D \backslash \bar{D}_{0}}^{2}+k^{4}\|\mathbf{u}\|_{D}^{2}
$$

Using

$\left\|\operatorname{curl} \operatorname{curl} \mathbf{u}-k^{2} \mathbf{u}\right\|_{D \backslash \bar{D}_{0}}^{2} \geq\|\operatorname{curl} \operatorname{curl} \mathbf{u}\|_{D \backslash \bar{D}_{0}}^{2}-2 k^{2}\|\operatorname{curl} \operatorname{curl} \mathbf{u}\|\left\|_{D \backslash \bar{D}_{0}}\left|\|\mathbf{u}\|_{D \backslash \bar{D}_{0}}+k^{4}\|\mathbf{u}\|\right|_{D \backslash \bar{D}_{0}}^{2}\right.$ we obtain

$\mathcal{A}_{k}(\mathbf{u}, \mathbf{u}) \geq \gamma\|\operatorname{curl} \operatorname{curl} \mathbf{u}\|_{D \backslash \bar{D}_{0}}^{2}-\left.\left.2 \gamma k^{2}\|\operatorname{curl} \operatorname{curl} \mathbf{u}\|\right|_{D \backslash \bar{D}_{0}}\|\mathbf{u}\|\right|_{D \backslash \bar{D}_{0}}+k^{4}(1+\gamma)\|\mathbf{u}\|_{D \backslash \bar{D}_{0}}^{2}+k^{4}\|\mathbf{u}\|_{D_{0}}^{2}$.

Using for example the identity

$$
\gamma X^{2}-2 \gamma X Y+(1+\gamma) Y^{2}=\left(\gamma+\frac{1}{2}\right)\left(Y-\frac{\gamma}{\gamma+\frac{1}{2}} X\right)^{2}+\frac{1}{2} Y^{2}+\frac{\gamma}{1+2 \gamma} X^{2}
$$


Setting $\tilde{\gamma}=\frac{\gamma}{1+2 \gamma}$ we deduce that

$$
\begin{aligned}
\mathcal{A}_{k}(\mathbf{u}, \mathbf{u}) & \geq \tilde{\gamma}\left(\|\operatorname{curl} \operatorname{curl} \mathbf{u}\|_{D \backslash \bar{D}_{0}}^{2}+k^{4}\|\mathbf{u}\|_{D \backslash \bar{D}_{0}}^{2}\right)+k^{4}\|\mathbf{u}\|_{D_{0}}^{2} \\
& \geq \tilde{\gamma}\left(\|\operatorname{curl} \operatorname{curl} \mathbf{u}\|_{D \backslash \bar{D}_{0}}^{2}+k^{4}\|\mathbf{u}\|_{D \backslash \bar{D}_{0}}^{2}+2 k^{4}\|\mathbf{u}\| \|_{D_{0}}^{2}\right)
\end{aligned}
$$

Since curl curl $\mathbf{u}=k^{2} \mathbf{u}$ in $D_{0}$ one also has that

$$
\mathcal{A}_{k}(\mathbf{u}, \mathbf{u}) \geq \tilde{\gamma}\left(\|\operatorname{curl} \operatorname{curl} \mathbf{u}\|_{D}^{2}+k^{4}\|\mathbf{u}\|_{D}^{2}\right)
$$

Integrating by parts, one has the following identity valid for all $\mathbf{u} \in V_{0}\left(D, D_{0}, k\right)$ :

$$
\left\|\operatorname{curl} \operatorname{curl} \mathbf{u}-k^{2} \mathbf{u}\right\|_{D \backslash \bar{D}_{0}}^{2}=\|\operatorname{curl} \operatorname{curl} \mathbf{u}\|_{D}^{2}+k^{4}\|\mathbf{u}\|_{D}^{2}-2 k^{2}\|\operatorname{curl} \mathbf{u}\|_{D}^{2} .
$$

Hence,

$$
2 k^{2}\|\operatorname{curl} \mathbf{u}\|_{D}^{2} \leq\|\operatorname{curl} \operatorname{curl} \mathbf{u}\|_{D}^{2}+k^{4}\|\mathbf{u}\|_{D}^{2} .
$$

Let $c_{k}=\min \left(\frac{k^{2}}{1+k^{2}}, \frac{k^{4}}{1+k^{2}}\right)$. Using (1.20) and (1.21) we obtain that

$$
\begin{aligned}
\mathcal{A}_{k}(\mathbf{u}, \mathbf{u}) & \geq \tilde{\gamma}\left(c_{k} \frac{1+k^{2}}{k^{2}}\|\operatorname{curl} \operatorname{curl} \mathbf{u}\|_{D}^{2}+c_{k}\left(1+k^{2}\right)\|\mathbf{u}\|_{D}^{2}\right) \\
& \geq c_{k} \tilde{\gamma}\left(\|\operatorname{curl} \operatorname{curl} \mathbf{u}\|_{D}^{2}+\|\mathbf{u}\|_{D}^{2}+\frac{1}{k^{2}}\left(\|\operatorname{curl} \operatorname{curl} \mathbf{u}\|_{D}^{2}+k^{4}\|\mathbf{u}\|_{D}^{2}\right)\right) \\
& \geq c_{k} \tilde{\gamma}\|\mathbf{u}\|_{\mathcal{U}(D)}^{2}
\end{aligned}
$$

Therefore $\mathcal{A}_{k}$ is a coercive sesquilinear form on $V_{0}\left(D, D_{0}, k\right) \times V_{0}\left(D, D_{0}, k\right)$. Moreover $\mathcal{B}_{k}$ defines a compact perturbation of $\mathcal{A}_{k}$ (see [10] lemma 3.4). The result now follows from the application of the Fredholm alternative.

Now assume that $(I-N)^{-1}$ is bounded positive definite. The variational formulation can also be written as

$$
\mathcal{A}_{k}^{\prime}\left(\mathbf{F}_{0}, \Psi\right)+\mathcal{B}_{k}\left(\mathbf{F}_{0}, \Psi\right)=-\mathcal{A}_{k}^{\prime}(\boldsymbol{\Theta}, \Psi)-\mathcal{B}_{k}(\boldsymbol{\Theta}, \Psi)
$$

with

$$
\begin{aligned}
\mathcal{A}_{k}^{\prime}(\mathbf{u}, \mathbf{v}):=\int_{D \backslash \bar{D}_{0}} N(I-N)^{-1} & \left(\operatorname{curl} \operatorname{curl} \mathbf{u}-k^{2} \mathbf{u}\right) \cdot\left(\operatorname{curl} \operatorname{curl} \overline{\mathbf{v}}-k^{2} \overline{\mathbf{v}}\right) d x \\
& +\int_{D \backslash \bar{D}_{0}} \operatorname{curl} \operatorname{curl} \mathbf{u} \cdot \operatorname{curl} \operatorname{curl} \overline{\mathbf{v}} d x+k^{4} \int_{D_{0}} \mathbf{u} \cdot \overline{\mathbf{v}} d x .
\end{aligned}
$$

Since $(I-N)^{-1}$ is bounded positive definite, there exists a constant $\gamma$ such that

$$
\begin{aligned}
\mathcal{A}_{k}^{\prime}(\mathbf{u}, \mathbf{u}) \geq(\gamma+1)\|\operatorname{curl} \operatorname{curl} \mathbf{u}\|_{D \backslash \bar{D}_{0}}^{2}-2 \gamma k^{2}\|\operatorname{curl} \operatorname{curl} \mathbf{u}\|\left\|_{D \backslash \bar{D}_{0}}\right\| \mathbf{u}\|\|_{D \backslash \bar{D}_{0}} \\
+\gamma k^{4}\|\mathbf{u}\|_{D \backslash \bar{D}_{0}}^{2}+k^{4}\|\mathbf{u}\|_{D_{0}}^{2}
\end{aligned}
$$

Then the same method as in the previous case from (1.19) show that $\mathcal{A}_{k}^{\prime}$ is a coercive sesquilinear form on $V_{0}\left(D, D_{0}, k\right) \times V_{0}\left(D, D_{0}, k\right)$ and the result follows from the Fredholm alternative. 
2. Transmission eigenvalues. We now study the homogeneous interior transmission problem and transmission eigenvalues as defined in Definition 1.2.

We first note that $k$ is a transmission eigenvalue if and only if the homogeneous problem

$$
\mathcal{A}_{k}\left(\mathbf{F}_{0}, \boldsymbol{\Psi}\right)+\mathcal{B}_{k}\left(\mathbf{F}_{0}, \Psi\right)=0 \text { for all } \boldsymbol{\Psi} \in V_{0}\left(D, D_{0}, k\right)
$$

has a nontrivial solution $\mathbf{F}_{0} \in V_{0}\left(D, D_{0}, k\right)$. Taking $\mathbf{\Psi}=\mathbf{F}_{0}$ we obtain

$$
\begin{array}{r}
\int_{D \backslash \bar{D}_{0}}(N-I)^{-1}\left(\operatorname{curl} \operatorname{curl} \mathbf{F}_{0}-k^{2} \mathbf{F}_{0}\right) \cdot\left(\operatorname{curl} \operatorname{curl} \overline{\mathbf{F}}_{0}-k^{2} \overline{\mathbf{F}}_{0}\right) d x+k^{4} \int_{D \backslash \bar{D}_{0}}\left|\mathbf{F}_{0}\right|^{2} d x \\
-k^{2} \int_{D \backslash \bar{D}_{0}}\left|\operatorname{curl} \mathbf{F}_{0}\right|^{2} d x+k^{2} \int_{\partial D_{0}} \nu \times \overline{\mathbf{F}}_{0} \cdot \operatorname{curl} \mathbf{F}_{0} d s=0
\end{array}
$$

In order to study transmission eigenvalues it suffices to study (2.1).

TheOREM 2.1. If $N \in L^{\infty}\left(D, \mathbb{R}^{3 \times 3}\right)$ is such that $N=I$ in $D_{0}$ and $\operatorname{Im}(N)$ is positive definite almost everywhere in $D \backslash \bar{D}_{0}$ then there are no transmission eigenvalues.

Proof. Using the fact that $\mathbf{F}_{0} \in V_{0}\left(D, D_{0}, k\right)$ and in particular curlcurl $\mathbf{F}_{0}-$ $k^{2} \mathbf{F}_{0}=0$ in $D_{0}$, we can re-write $(2.1)$ as

$$
\begin{aligned}
\int_{D \backslash \bar{D}_{0}}(N-I)^{-1}\left(\operatorname{curl} \operatorname{curl} \mathbf{F}_{0}-\right. & \left.k^{2} \mathbf{F}_{0}\right) \\
& \cdot\left(\operatorname{curl} \operatorname{curl} \overline{\mathbf{F}}_{0}-k^{2} \overline{\mathbf{F}}_{0}\right) d x \\
& +k^{4} \int_{D}\left|\mathbf{F}_{0}\right|^{2} d x-k^{2} \int_{D}\left|\operatorname{curl} \mathbf{F}_{0}\right|^{2} d x=0 .
\end{aligned}
$$

Since $\operatorname{Im}\left((N-I)^{-1}\right)$ is negative definite in $D \backslash \bar{D}_{0}$ and all the terms in the above equation are real except for the first one, by taking the imaginary part we deduce that $\mathbf{F}_{0}$ satisfies Maxwell's equation in $D \backslash \bar{D}_{0}$ and then in all $D$. Since $\mathbf{F}_{0}$ has zero Cauchy data on $\partial D$ we obtain $\mathbf{F}_{0}=0$ in $D$ and therefore $k$ is not a transmission eigenvalue.

In the following, we shall assume that $\operatorname{Im}(N)=0$.

2.1. Discreteness of the set of transmission eigenvalues. The proof of the discretness of the set of transmission eigenvalues relies on the analytic Fredholm theory. This theory will guarantee that the operator associated with the resolution of the interior transmission problem is injective except for at most a discrete set of values of $k$. Thus we first show that this operator is analytic with respect to $k \in \mathbb{C}$ in some neighborhood of the real axis.

Finding transmission eigenvalues is equivalent to finding $k>0$ such that the problem

$$
\mathcal{A}_{k}(\mathbf{E}, \Psi)+\mathcal{B}_{k}(\mathbf{E}, \Psi)=0 \text { for all } \boldsymbol{\Psi} \in V_{0}\left(D, D_{0}, k\right)
$$

has non trivial solutions $\mathbf{E} \in V_{0}\left(D, D_{0}, k\right)$. This is equivalent to finding the values of $k$ for which

$$
A_{k}+B_{k}: V_{0}\left(D, D_{0}, k\right) \longrightarrow V_{0}\left(D, D_{0}, k\right)
$$


has a nontrivial kernel, where $A_{k}$ is the positive definite self-adjoint operator associated with the coercive bilinear form $\mathcal{A}_{k}(\cdot, \cdot)$ and $B_{k}$ is the compact operator associated with the bilinear form $\mathcal{B}_{k}(\cdot, \cdot)$.

To avoid dealing with function spaces depending on $k$ we shall make use of an analytic operator $\tilde{P}_{k}$ from $\mathcal{U}_{0}(D)$ into $V_{0}\left(D, D_{0}, k\right)$ in order to build an analytic extension of $A_{k}$ and $B_{k}$ with operators acting on $\mathcal{U}_{0}(D)$.

Let $k \in \mathbb{C}$. For $\mathbf{E} \in \mathcal{U}_{0}(D)$, we define $\theta_{k} \mathbf{E}$ by

$$
\left(\theta_{k} \mathbf{E}\right)(x)=\int_{D_{0}}\left(\operatorname{curl} \operatorname{curl} \mathbf{E}-k^{2} \mathbf{E}\right)(y) \mathbb{G}(x, y) d y
$$

with $\mathbb{G}(x, y)=y_{0}(k|x-y|) I+\frac{1}{k^{2}} \nabla_{x} \operatorname{div}_{x}\left(y_{0}(k|x-y|) I\right)$ where $y_{0}(t)=k \frac{\cos (t)}{4 \pi t}$. One has in particular

$$
\operatorname{curl}_{x} \operatorname{curl}_{x} \mathbb{G}(x, y)-k^{2} \mathbb{G}(x, y)=\delta_{y} I .
$$

Using the regularity of the volume potential we have that $\theta_{k} \mathbf{E} \in L^{2}(D)^{3}$. Moreover since

$$
\operatorname{curl}_{x} \mathbb{G}(x, y)=\operatorname{curl}_{x}\left(y_{0}(k|x-y|) I\right)
$$

we have

$$
\operatorname{curl}\left(\theta_{k} \mathbf{E}\right)(x)=\operatorname{curl}_{x} \int_{D_{0}}\left(\operatorname{curl} \operatorname{curl} \mathbf{E}-k^{2} \mathbf{E}\right)(y) y_{0}(k|x-y|) I d y, \quad x \in D .
$$

Once again using regularity of volume potential we obtain that $\operatorname{curl}\left(\theta_{k} \mathbf{E}\right) \in H^{1}(D)$. Therefore $\theta_{k} \mathbf{E} \in \mathcal{U}_{0}(D)$ and there exists a constant $C(k)$ such that

$$
\left\|\theta_{k} \mathbf{E}\right\|_{\mathcal{U}(D)} \leq C(k) \| \operatorname{curl} \operatorname{curl} \mathbf{E}-\left.k^{2} \mathbf{E}\right|_{L^{2}\left(D_{0}\right)} .
$$

$\theta_{k}: \mathcal{U}_{0}(D) \longrightarrow \mathcal{U}_{0}(D)$ depends analytically with respect to $k \in \mathbb{C}$.

Let $\chi$ be a cutoff function that equals 1 in $D_{0}$ and 0 outside $D$. We define the continuous operator $\tilde{P}_{k}: \mathcal{U}_{0}(D) \longrightarrow \mathcal{U}_{0}(D)$ by :

$$
\tilde{P}_{k} \mathbf{E}:=\mathbf{E}-\chi \theta_{k} \mathbf{E} .
$$

We first observe that for $\mathbf{E} \in V_{0}\left(D, D_{0}, k\right)$ we have

$$
\theta_{k} \mathbf{E}=0 \text { and } \tilde{P}_{k} \mathbf{E}=\mathbf{E} \text {. }
$$

Moreover since

$$
\operatorname{curl} \operatorname{curl} \theta_{k} \mathbf{E}-k^{2} \theta_{k} \mathbf{E}=\operatorname{curl} \operatorname{curl} \mathbf{E}-k^{2} \mathbf{E} \text { in } D_{0},
$$

we also have $\tilde{P}_{k} \mathbf{E} \in V_{0}\left(D, D_{0}, k\right)$ for all $\mathbf{E} \in \mathcal{U}_{0}(D)$. Finally, from the analyticity of $\theta_{k}, \tilde{P}_{k}$ also depends analytically on complex $k$ with positive real part.

Using the Riesz representation theorem, we now introduce the operators $\tilde{A}_{k}$ and $\tilde{B}_{k}$ from $\mathcal{U}_{0}(D)$ into $\mathcal{U}_{0}(D)$ defined by

$$
\begin{aligned}
\left(\tilde{A}_{k} \mathbf{E}, \mathbf{F}\right)_{\mathcal{U}(D)} & =\mathcal{A}_{k}\left(\tilde{P}_{k} \mathbf{E}, \overline{\tilde{P}_{k} \overline{\mathbf{F}}}\right)+\alpha\left(\theta_{k} \mathbf{E}, \overline{\theta_{k} \overline{\mathbf{F}}}\right)_{\mathcal{U}(D)} \\
\left(\tilde{B}_{k} \mathbf{E}, \mathbf{F}\right)_{\mathcal{U}(D)} & =\mathcal{B}_{k}\left(\tilde{P}_{k} \mathbf{E}, \overline{\tilde{P}_{k} \overline{\mathbf{F}}}\right)
\end{aligned}
$$


for all $\mathbf{E}, \mathbf{F}$ in $\mathcal{U}_{0}(D)$ where $\alpha$ is a positive constant that will be fixed later (and is independent of $k$ ). The analyticity of $\tilde{P}_{k}$ and $\theta_{k}$ as well as the expression of $\mathcal{A}_{k}$ and $\mathcal{B}_{k}$ show that $\tilde{A}_{k}$ and $\tilde{B}_{k}$ depend analytically on $k \in \mathbb{C}$ with $\operatorname{Re}(k)>0$. Moreover the operator $\tilde{B}_{k}$ is compact.

Observe that if $k$ is real, for $\mathbf{E} \in V_{0}\left(D, D_{0}, k\right)$ then we have $\overline{\mathbf{E}} \in V_{0}\left(D, D_{0}, k\right)$ and hence from (2.4), we have that

$$
\tilde{A}_{k} \mathbf{E}=A_{k} \mathbf{E}, \quad \tilde{B}_{k} \mathbf{E}=B_{k} \mathbf{E} \quad \forall \mathbf{E} \in V_{0}\left(D, D_{0}, k\right) \text { and } \forall k \in \mathbb{R} .
$$

Hence we conclude that for real $k$, if $A_{k}+B_{k}$ is not injective then $\tilde{A}_{k}+\tilde{B}_{k}$ is not injective. In order to show that the set of transmission eigenvalues is at most discrete it is sufficient to prove that the set of $k$ for which $\tilde{A}_{k}+\tilde{B}_{k}$ is not injective is at most discrete. For that purpose we shall prove the following lemma :

Lemma 2.2. Let $k$ be positive and real. Then

1. There exists $\alpha_{0}$ independent from $k$ such that for all $\alpha \geq \alpha_{0}$ the operator $\tilde{A}_{k}$ is strictly coercive for all $k>0$

2. There exists $k_{0}$ such that for all $0<k \leq k_{0}$ the operator $\tilde{A}_{k}+\tilde{B}_{k}$ is injective.

Proof. Assume that $k$ is real. Therefore

$$
\left(\tilde{A}_{k} \mathbf{E}, \mathbf{E}\right)_{\mathcal{U}(D)}=\mathcal{A}_{k}\left(\tilde{P}_{k} \mathbf{E}, \overline{\tilde{P}_{k} \overline{\mathbf{E}}}\right)+\alpha\left\|\theta_{k} \mathbf{E}\right\|_{\mathcal{U}(D)}^{2} .
$$

From the coercivity of $\mathcal{A}_{k}$ on $V_{0}\left(D, D_{0}, k\right)$ we have that

$$
\left(\tilde{A}_{k} \mathbf{E}, \mathbf{E}\right)_{\mathcal{U}(D)} \geq \gamma_{k}\left\|\tilde{P}_{k} \mathbf{E}\right\|_{\mathcal{U}(D)}^{2}+\alpha\left\|\theta_{k} \mathbf{E}\right\|_{\mathcal{U}(D)}^{2}
$$

where $\gamma_{k}=c_{k} \tilde{\gamma}$ with $c_{k}<1$ and $\tilde{\gamma}$ depending only on $N$. From the expression of $\tilde{P}_{k} \mathbf{E}$ one sees that there exists a constant $c$ that depends only on $\chi$ such that

$$
\begin{gathered}
\left\|\tilde{P}_{k} \mathbf{E}\right\|_{\mathcal{U}(D)}^{2} \geq\|\mathbf{E}\|_{\mathcal{U}(D)}^{2}-2 c|| \mathbf{E}\left\|_{\mathcal{U}(D)}\right\| \theta_{k} \mathbf{E}\left\|_{\mathcal{U}(D)}+\right\| \chi \theta_{k} \mathbf{E} \|_{\mathcal{U}^{(D)}}^{2} \\
\left(\tilde{A}_{k} \mathbf{E}, \mathbf{E}\right)_{\mathcal{U}(D)} \geq \gamma_{k}\|\mathbf{E}\|_{\mathcal{U}_{(D)}}^{2}-2 c \gamma_{k}\|\mathbf{E}\|_{\mathcal{U}(D)}\left\|\theta_{k} \mathbf{E}\right\|_{\mathcal{U}(D)}+\alpha\left\|\theta_{k} \mathbf{E}\right\|_{\mathcal{U}(D)}^{2} .
\end{gathered}
$$

Let $\alpha_{0}=\tilde{\gamma} c^{2}$. Since $\gamma_{k}<\tilde{\gamma}$, we observe that $\left(\gamma_{k} c\right)^{2}<\gamma_{k} \alpha$ for all $k$ and $\alpha \geq \alpha_{0}$. Therefore we can conclude that the operator $\tilde{A}_{k}$ is strictly coercive for $\alpha \geq \alpha_{0}$.

We now prove the second assertion. We observe that

$$
\left(\tilde{A}_{k} \mathbf{E}, \mathbf{E}\right)_{\mathcal{U}(D)}+\left(\tilde{B}_{k} \mathbf{E}, \mathbf{E}\right)_{\mathcal{U}(D)}=\mathcal{A}_{k}\left(\tilde{P}_{k} \mathbf{E}, \overline{\tilde{P}_{k} \mathbf{E}}\right)+\alpha\left\|\theta_{k} \mathbf{E}\right\|_{\mathcal{U}(D)}^{2}+\mathcal{B}_{k}\left(\tilde{P}_{k} \mathbf{E}, \overline{\tilde{P}_{k} \mathbf{E}}\right) .
$$

Therefore $\left(\tilde{A}_{k} \mathbf{E}, \mathbf{E}\right)_{\mathcal{U}(D)}+\left(\tilde{B}_{k} \mathbf{E}, \mathbf{E}\right)_{\mathcal{U}(D)}=0$ implies that $\theta_{k} \mathbf{E}=0$ and

$$
\mathcal{A}_{k}\left(\tilde{P}_{k} \mathbf{E}, \tilde{P}_{k} \mathbf{E}\right)+\mathcal{B}_{k}\left(\tilde{P}_{k} \mathbf{E}, \tilde{P}_{k} \mathbf{E}\right)=0
$$

To show that $\tilde{P}_{k} \mathbf{E}=0$ and conclude the proof, we need the following theorem:

Theorem 2.3. Let $N \in L^{\infty}\left(D, \mathbb{R}^{3 \times 3}\right)$. Let $0<\eta_{1}(x)<\eta_{2}(x)<\eta_{3}(x)$ be the eigenvalues of the positive definite matrix $N$. We denote by $N^{*}=\sup _{D \backslash \bar{D}_{0}} \eta_{3}(x)$ and $N_{*}=\inf _{D \backslash \bar{D}_{0}} \eta_{1}(x)$. If $k$ is a transmission eigenvalue then

$$
k^{2}>\frac{\lambda_{0}(D)}{N^{*}} \text { if }(N-I)^{-1} \text { is bounded positive definite, }
$$


or

$$
k^{2}>\lambda_{0}(D) \text { if }(I-N)^{-1} \text { is bounded positive definite, }
$$

where $\lambda_{0}(D)$ is the first Dirichlet eigenvalue of $-\Delta$ in $D$.

Proof. We want to show that if $k>0$ is sufficiently small then $k$ is not a transmission eigenvalue. It suffices to show that for $k>0$ sufficiently small, if $\mathbf{u} \in V_{0}\left(D, D_{0}, k\right)$ satisfies (2.2) then $\mathbf{u}$ is zero.

We first assume that $(N-I)^{-1}$ is bounded positive definite. In order to find lower bound for the first transmission eigenvalue we study $(2.2)$. For $\gamma=\frac{1}{N^{*}-1}$ we have

$\mathcal{A}_{k}(\mathbf{u}, \mathbf{u}) \geq \gamma\|\operatorname{curl} \operatorname{curl} \mathbf{u}\|_{D \backslash \bar{D}_{0}}^{2}-\left.2 \gamma k^{2}\|\operatorname{curl} \operatorname{curl} \mathbf{u}\|\right|_{D \backslash \bar{D}_{0}}\|\mathbf{u}\|\left\|_{D \backslash \bar{D}_{0}}+k^{4}(1+\gamma)\right\| \mathbf{u}\left\|_{D \backslash \bar{D}_{0}}^{2}+k^{4}\right\| \mathbf{u} \|\left.\right|_{D_{0}} ^{2}$.

From the identity

$$
\gamma X^{2}-2 \gamma X Y+(1+\gamma) Y^{2}=\epsilon\left(Y-\frac{\gamma}{\epsilon} X\right)^{2}+\left(\gamma-\frac{\gamma^{2}}{\epsilon}\right) X^{2}+(1+\gamma-\epsilon) Y^{2}
$$

with $X=\|\operatorname{curl} \operatorname{curl} \mathbf{u}\|_{D \backslash \bar{D}_{0}}$ and $Y=\|\mathbf{u}\|_{D \backslash \bar{D}_{0}}$ we deduce that

$$
\mathcal{A}_{k}(\mathbf{u}, \mathbf{u}) \geq\left(\gamma-\frac{\gamma^{2}}{\epsilon}\right)\|\operatorname{curl} \operatorname{curl} \mathbf{u}\|_{D \backslash \bar{D}_{0}}^{2}+k^{4}(1+\gamma-\epsilon)\|\mathbf{u}\|_{D \backslash \bar{D}_{0}}^{2}+k^{4}\|\mathbf{u}\|_{D_{0}}^{2} .
$$

Using $k^{2} \mathbf{u}=$ curl curl $\mathbf{u}$ in $D_{0}$ and $1 \geq\left(\gamma-\frac{\gamma^{2}}{\epsilon}\right)+(1+\gamma-\epsilon)$ we obtain

$\mathcal{A}_{k}(\mathbf{u}, \mathbf{u})+\mathcal{B}_{k}(\mathbf{u}, \mathbf{u}) \geq\left(\gamma-\frac{\gamma^{2}}{\epsilon}\right)\|\operatorname{curl} \operatorname{curl} \mathbf{u}\|_{D}^{2}+k^{4}(1+\gamma-\epsilon)\|\mathbf{u}\|_{D}^{2}-k^{2}\|\operatorname{curl} \mathbf{u}\|_{D}^{2}$.

Moreover, for all $\mathbf{u} \in \mathcal{U}_{0}(D)$ we have the following inequality (see [6]) :

$$
\|\operatorname{curl} \mathbf{u}\|_{L^{2}(D)}^{2} \leq \frac{1}{\lambda_{0}(D)}\|\operatorname{curl} \operatorname{curl} \mathbf{u}\|_{L^{2}(D)}^{2}
$$

Then using (2.9) we get

$$
\mathcal{A}_{k}(\mathbf{u}, \mathbf{u})+\mathcal{B}_{k}(\mathbf{u}, \mathbf{u}) \geq\left(\gamma-\frac{\gamma^{2}}{\epsilon}-\frac{k^{2}}{\lambda_{0}(D)}\right)\|\operatorname{curl} \operatorname{curl} \mathbf{u}\|_{D}^{2}+k^{4}(1+\gamma-\epsilon)\|\mathbf{u}\|_{D}^{2}
$$

Hence letting $\epsilon$ arbitrary close to $\gamma+1, k$ is not a transmission eigenvalue if

$$
k^{2} \leq \frac{\lambda_{0}(D)}{N^{*}}
$$

Now we prove the result when $(I-N)^{-1}$ is bounded positive definite. We have the following equality :

$$
\begin{aligned}
& \mathcal{A}_{k}^{\prime}(\mathbf{u}, \mathbf{v})+\mathcal{B}_{k}(\mathbf{u}, \mathbf{v})=\int_{D \backslash \bar{D}_{0}} N(I-N)^{-1}\left(\operatorname{curl} \operatorname{curl} \mathbf{u}-k^{2} \mathbf{u}\right) \cdot\left(\operatorname{curl} \operatorname{curl} \overline{\mathbf{v}}-k^{2} \overline{\mathbf{v}}\right) d x \\
& +k^{4} \int_{D_{0}} \mathbf{u} \cdot \overline{\mathbf{v}} d x+\int_{D \backslash \bar{D}_{0}} \operatorname{curl} \operatorname{curl} \mathbf{u} \cdot \operatorname{curl} \operatorname{curl} \overline{\mathbf{v}} d x-k^{2} \int_{D} \operatorname{curl} \mathbf{u} \cdot \operatorname{curl} \overline{\mathbf{v}} d x .
\end{aligned}
$$


For $\gamma=\frac{N_{*}}{1-N_{*}}$, using $(2.8)$ and (2.9) we have

$$
\begin{aligned}
\mathcal{A}_{k}(\mathbf{u}, \mathbf{u})+\mathcal{B}_{k}(\mathbf{u}, \mathbf{u}) \geq & (\gamma+1)\|\operatorname{curl} \operatorname{curl} \mathbf{u}\|_{D \backslash \bar{D}_{0}}^{2}-2 \gamma k^{2}\|\operatorname{curl} \operatorname{curl} \mathbf{u}\|_{D \backslash \bar{D}_{0}}\|\mathbf{u}\|_{D \backslash \bar{D}_{0}} \\
& +k^{4} \gamma\|\mathbf{u}\|_{D \backslash \bar{D}_{0}}^{2}+k^{4}\|\mathbf{u}\|_{D_{0}}^{2}-k^{2}\|\operatorname{curl} \mathbf{u}\|_{D}^{2} \\
\geq & (1+\gamma-\varepsilon)\left\|\left.\left.\operatorname{curl} \operatorname{curl} \mathbf{u}\right|^{2}\right|_{D}+\left(\gamma-\frac{\gamma^{2}}{\varepsilon}\right)\right\| \mathbf{u}\left\|_{D}^{2}-k^{2}\right\| \operatorname{curl} \mathbf{u} \|_{D}^{2} \\
\geq & \left(1+\gamma-\varepsilon-\frac{k^{2}}{\lambda_{0}(D)}\right)\|\operatorname{curl} \operatorname{curl} \mathbf{u}\|_{D}^{2}+\left(\gamma-\frac{\gamma^{2}}{\varepsilon}\right)\|\mathbf{u}\|_{D}^{2} .
\end{aligned}
$$

Hence letting $\varepsilon$ arbitrary close to $\gamma$, we deduce that $k$ cannot be a transmission eigenvalue if

$$
k^{2} \leq \lambda_{0}(D)
$$

End of the proof of Lemma 2.2: According to the previous theorem, if (2.5) holds for $k$ small enough then $\tilde{P}_{k} \mathbf{E}=0$. We conclude that $\mathbf{E}=\tilde{P}_{k} \mathbf{E}+\chi \theta_{k} \mathbf{E}=0$. Then for $k$ small enough, $\tilde{A}_{k}+\tilde{B}_{k}$ is injective.

THEOREM 2.4. Let $N \in L^{\infty}\left(D, \mathbb{R}^{3 \times 3}\right)$. Then the set of transmission eigenvalues is discrete.

Proof. The previous lemma shows in particular that for $\alpha$ sufficiently large $\tilde{A}_{k}$ is coercive in a neighborhood of the real axis (since $\tilde{A}_{k}$ is continuous with respect to $k$ ) and therefore invertible. In this neighborhood $\tilde{A}_{k}^{-1}$ is analytic and hence the operator $I+\tilde{A}_{k}^{-1} \tilde{B}_{k}$ depends analytically on $k$ and is injective for $k$ sufficiently small. The analytic Fredholm theory now shows that this operator is injective for all values of $k$ in this neighborhood except for at most a discrete set of values.

2.2. Existence of transmission eigenvalues. We observe that $k>0$ is a transmission eigenvalue if and only if the operator

$$
A_{k}+B_{k}: V_{0}\left(D, D_{0}, k\right) \longrightarrow V_{0}\left(D, D_{0}, k\right)
$$

has a nontrivial kernel, where $A_{k}$ is the positive definite self-adjoint operator associated with the coercive bilinear form $\mathcal{A}_{k}(\cdot, \cdot)$ and $B_{k}$ is the compact operator associated with the bilinear form $\mathcal{B}_{k}(\cdot, \cdot)$. Define the operator $A_{k}^{-1 / 2}$ by $A_{k}^{-1 / 2}=\int_{0}^{\infty} \lambda^{-1 / 2} d E_{\lambda}$ where $d E_{\lambda}$ is the spectral measure associated with the positive self-adjoint operator $A_{k}$. In particular, $A_{k}^{-1 / 2}$ is also bounded, positive definite and self-adjoint. Hence it is obvious that $k$ is a transmission eigenvalue if and only if the operator

$$
I_{k}+A_{k}^{-1 / 2} B_{k} A_{k}^{-1 / 2}: V_{0}\left(D, D_{0}, k\right) \longrightarrow V_{0}\left(D, D_{0}, k\right)
$$

has a nontrivial kernel. Note that $A_{k}^{-1 / 2} B_{k} A_{k}^{-1 / 2}$ is a compact self-adjoint operator. To avoid dealing with $k$-dependent function space $V_{0}\left(D, D_{0}, k\right)$ we consider

$$
I+R_{k} A_{k}^{-1 / 2} B_{k} A_{k}^{-1 / 2} P_{k}: \mathcal{U}_{0}(D) \longrightarrow \mathcal{U}_{0}(D)
$$

where $P_{k}: \mathcal{U}_{0}(D) \longrightarrow V_{0}\left(D, D_{0}, k\right)$ and $R_{k}: V_{0}\left(D, D_{0}, k\right) \longrightarrow \mathcal{U}_{0}(D)$ are respectively the orthogonal projection and the injection operator. We first have to show that $P_{k}$ is continuous from $\mathcal{U}_{0}(D)$ into $V_{0}\left(D, D_{0}, k\right)$. For this purpose we need the following lemma : 
LEMma 2.5. Assume $|k|<k_{0}$ for $k_{0}>0$. Then there exists a constant $C\left(k_{0}\right)$ such that

$$
\left\|\mathbf{E}-P_{k} \mathbf{E}\right\|_{\mathcal{U}_{0}(D)} \leq C\left(k_{0}\right)\left\|\operatorname{curl} \operatorname{curl} \mathbf{E}-k^{2} \mathbf{E}\right\|_{L^{2}\left(D_{0}\right)}
$$

for all $\mathbf{E} \in \mathcal{U}_{0}(D)$.

Proof. Let $\mathbf{E} \in \mathcal{U}_{0}(D)$ and let $\tilde{P}_{k}$ be the operator defined by (2.3). Then

$$
\begin{aligned}
\left\|P_{k} \mathbf{E}-\mathbf{E}\right\|_{\mathcal{U}_{0}(D)} & \leq\left\|\tilde{P}_{k} \mathbf{E}-\mathbf{E}\right\|_{\mathcal{U}_{0}(D)}=\left\|\chi \theta_{k} \mathbf{E}\right\|_{\mathcal{U}_{0}(D)} \\
& \leq C\left\|\theta_{k} \mathbf{E}\right\|_{\mathcal{U}_{0}(D)} \leq C C(k)\left\|\operatorname{curl} \operatorname{curl} \mathbf{E}-k^{2} \mathbf{E}\right\|_{L^{2}\left(D_{0}\right)} .
\end{aligned}
$$

Since $\theta_{k}$ depends continuously on $k$, one can bound $C C(k)$ by a constant that only depends on $k_{0}$ for all $k \leq k_{0}$.

THEOREM 2.6. The projection operator $P_{k}: \mathcal{U}_{0}(D) \longrightarrow V_{0}\left(D, D_{0}, k\right)$ is continuous with respect to $k>0$.

Proof. Let $k$ and $k^{\prime}$ be positive reals less than $k_{0}$ and let $\mathbf{E}$ be in $\mathcal{U}_{0}(D)$. Set $\mathbf{E}_{k}:=P_{k} \mathbf{E}$ and $\mathbf{E}_{k^{\prime}}:=P_{k^{\prime}} \mathbf{E}$. Then

$$
\left\|\mathbf{E}_{k}-\mathbf{E}_{k^{\prime}}\right\|_{\mathcal{U}_{0}(D)}^{2}=\left\|P_{k}\left(\mathbf{E}_{k}-\mathbf{E}_{k^{\prime}}\right)\right\|_{\mathcal{U}_{0}(D)}^{2}+\left\|\left(I-P_{k}\right)\left(\mathbf{E}_{k}-\mathbf{E}_{k^{\prime}}\right)\right\|_{\mathcal{U}_{0}(D)}^{2} .
$$

On the one hand using Lemma 2.5,

$$
\begin{aligned}
\left\|\left(I-P_{k}\right)\left(\mathbf{E}_{k}-\mathbf{E}_{k^{\prime}}\right)\right\|_{\mathcal{U}_{0}(D)} & =\left\|\left(I-P_{k}\right) \mathbf{E}_{k^{\prime}}\right\|_{\mathcal{U}_{0}(D)} \\
& \leq C\left(k_{0}\right)\left\|\operatorname{curl} \operatorname{curl} \mathbf{E}_{k^{\prime}}-k^{2} \mathbf{E}_{k^{\prime}}\right\|_{L^{2}\left(D_{0}\right)} \\
& =C\left(k_{0}\right)\left|k^{\prime 2}-k^{2}\left\|\mid \mathbf{E}_{k^{\prime}}\right\|_{L^{2}\left(D_{0}\right)}\right. \\
& \leq C\left(k_{0}\right)\left|k^{\prime 2}-k^{2}\|\mid \mathbf{E}\|_{\mathcal{U}_{0}(D)}\right.
\end{aligned}
$$

and on the other hand,

$$
\begin{aligned}
\left\|P_{k}\left(\mathbf{E}_{k}-\mathbf{E}_{k^{\prime}}\right)\right\|_{\mathcal{U}_{0}(D)}^{2} & =\left(P_{k}\left(\mathbf{E}_{k}-\mathbf{E}_{k^{\prime}}\right), P_{k}\left(\mathbf{E}_{k}-\mathbf{E}_{k^{\prime}}\right)\right)_{\mathcal{U}_{0}(D)} \\
& =\left(P_{k}\left(\mathbf{E}_{k}-\mathbf{E}_{k^{\prime}}\right), \mathbf{E}_{k}-\mathbf{E}_{k^{\prime}}\right)_{\mathcal{U}_{0}(D)} \\
& =\left(P_{k}\left(\mathbf{E}_{k}-\mathbf{E}_{k^{\prime}}\right), \mathbf{E}_{k}-\mathbf{E}+\mathbf{E}-\mathbf{E}_{k^{\prime}}\right)_{\mathcal{U}_{0}(D)} \\
& =\left(P_{k}\left(\mathbf{E}_{k}-\mathbf{E}_{k^{\prime}}\right), \mathbf{E}-\mathbf{E}_{k^{\prime}}\right)_{\mathcal{U}_{0}(D)} \\
& =\left(\left(I-P_{k^{\prime}}\right) P_{k}\left(\mathbf{E}_{k}-\mathbf{E}_{k^{\prime}}\right), \mathbf{E}\right)_{\mathcal{U}_{0}(D)}
\end{aligned}
$$

Applying Lemma 2.5, we have

$$
\begin{aligned}
\left\|\left(I-P_{k^{\prime}}\right) P_{k}\left(\mathbf{E}_{k}-\mathbf{E}_{k^{\prime}}\right)\right\|_{\mathcal{U}_{0}(D)} & \leq C\left(k_{0}\right)\left\|\left(\operatorname{curl} \operatorname{curl}-k^{\prime 2}\right) P_{k}\left(\mathbf{E}_{k}-\mathbf{E}_{k^{\prime}}\right)\right\|_{L^{2}\left(D_{0}\right)} \\
& =C\left(k_{0}\right)\left|k^{\prime 2}-k^{2}\left\|\mid P_{k}\left(\mathbf{E}_{k}-\mathbf{E}_{k^{\prime}}\right)\right\|_{L^{2}\left(D_{0}\right)}\right. \\
& \leq C\left(k_{0}\right)\left|k^{\prime 2}-k^{2}\left\|\mid \mathbf{E}_{k}-\mathbf{E}_{k^{\prime}}\right\|_{\mathcal{U}_{0}(D)} .\right.
\end{aligned}
$$

Therefore, from (2.14) and (2.13), we have

$$
\left\|P_{k}\left(\mathbf{E}_{k}-\mathbf{E}_{k^{\prime}}\right)\right\|_{\mathcal{U}_{0}(D)}^{2} \leq C\left(k_{0}\right)\left|k^{\prime 2}-k^{2}\left\|\mid \mathbf{E}_{k}-\mathbf{E}_{k^{\prime}}\right\|_{\mathcal{U}_{0}(D)}\|\mathbf{E}\|_{\mathcal{U}_{0}(D)} .\right.
$$

Using the previous estimates in the first equality yields

$$
\left\|\mathbf{E}_{k}-\mathbf{E}_{k^{\prime}}\right\|_{\mathcal{U}_{0}(D)} \leq \frac{\sqrt{5}+1}{2} C\left(k_{0}\right) \mid k^{\prime 2}-k^{2}\|\mathbf{E}\|_{\mathcal{U}_{0}(D)}
$$


which proves in particular that $k \longmapsto P_{k} \mathbf{E}$ is continuous.

We can show that the mapping $k \longmapsto R_{k} A_{k}^{-1 / 2} B_{k} A_{k}^{-1 / 2} P_{k}$ is continuous for $k>0$. Therefore, from the min-max principle for the eigenvalues $\lambda(k)$ of the compact and self-adjoint operator $R_{k} A_{k}^{-1 / 2} B_{k} A_{k}^{-1 / 2} P_{k}$ we can conclude that $\lambda(k)$ is a continuous function of $k$.

The proof of the existence of transmission eigenvalues is based on the following theorem proven in [6] :

Theorem 2.7. Let $T_{k}:=R_{k} A_{k}^{-1 / 2} B_{k} A_{k}^{-1 / 2} P_{k}$. Assume that :

(i) There is a $k_{1}$ such that $I+T_{k_{1}}$ is positive on $\mathcal{U}_{0}(D)$

(ii) There is a $k_{2}>k_{1}$ such that $I+T_{k_{2}}$ is non positive on a p-dimensional subspace $W_{k}$ of $\mathcal{U}_{0}(D)$.

Then there are $p$ transmission eigenvalues in $\left[k_{1}, k_{2}\right]$ counting their multiplicity.

The multiplicity of a transmission eigenvalue $k_{0}$ is defined as the dimension of the kernel of $I+T_{k_{0}}$.

Let $r>0$. We denote by $k_{0}(r, n)$ the first transmission eigenvalue for a ball of radius $r$ and $N=n I$ (see [7] for the existence of such eigenvalues). Let $M(r)$ be the maximum number of two by two disjoint balls of radius $r$ that can be inserted in $D \backslash \bar{D}_{0}$.

Theorem 2.8. For all $r>0$, there exist at least $M(r)$ transmission eigenvalues in

1. $\left[\frac{\lambda_{0}(D)}{N_{*}}, k_{0}\left(r, N_{*}\right)\right]$ if $(N-I)^{-1}$ is bounded positive definite,

2. $\left[\lambda_{0}(D), k_{0}\left(r, N^{*}\right)\right]$ if $(I-N)^{-1}$ is bounded positive definite.

Proof. Assume first that $(N-I)^{-1}$ is bounded positive definite. From theorem 2.3 , we know that $A_{k_{1}}+B_{k_{1}}$ is positive for $k_{1}:=\frac{\lambda_{0}(D)}{N^{*}}$.

Next we look for $k_{2}>k_{1}$ such that $I+T_{k_{2}}$ is non positive on a p-dimensional subspace of $\mathcal{U}_{0}(D)$. We first notice that for all $k>0$ :

$$
\begin{aligned}
\left(A_{k} \mathbf{u}+B_{k} \mathbf{u}, \mathbf{u}\right)= & \int_{D \backslash \bar{D}_{0}}(N-I)^{-1}\left(\operatorname{curl} \operatorname{curl} \mathbf{u}-k^{2} \mathbf{u}\right) \cdot\left(\operatorname{curl} \operatorname{curl} \overline{\mathbf{u}}-k^{2} \overline{\mathbf{u}}\right) d x \\
& -k^{2} \int_{D}|\operatorname{curl} \mathbf{u}|^{2} d x+k^{4} \int_{D}|\mathbf{u}|^{2} d x \\
\leq & \frac{1}{N_{*}-1} \int_{D \backslash \bar{D}_{0}}\left|\operatorname{curl} \operatorname{curl} \mathbf{u}-k^{2} \mathbf{u}\right|^{2} d x-k^{2} \int_{D}|\operatorname{curl} \mathbf{u}|^{2} d x+k^{4} \int_{D}|\mathbf{u}|^{2} d x .
\end{aligned}
$$

Let $C_{j}, j=1 \ldots M(r)$ be $M(r)$ two by two disjoint balls included in $D \backslash \bar{D}_{0}$. For each ball $C_{j}$, there exists an eigenvector $\mathbf{u}_{j} \in \mathcal{U}_{0}\left(C_{j}\right)$ corresponding to the transmission eigenvalue $k_{2}:=k_{0}\left(r, N_{*}\right)$ which satisfies the variational formulation of the corresponding interior transmission problem :

$$
\frac{1}{N_{*}-1} \int_{C_{j}}\left|\operatorname{curl} \operatorname{curl} \mathbf{u}_{j}-k_{2}^{2} \mathbf{u}_{j}\right|^{2} d x-k_{2}^{2} \int_{C_{j}}\left|\operatorname{curl} \mathbf{u}_{j}\right|^{2} d x+k_{2}^{4} \int_{C_{j}}\left|\mathbf{u}_{j}\right|^{2} d x=0 .
$$

We still denote by $\mathbf{u}_{j}$ the extension of $\mathbf{u}_{j}$ by 0 to all $D$. Then $\mathbf{u}_{j} \in V_{0}\left(D, D_{0}, k\right)$ and

$$
\frac{1}{N_{*}-1} \int_{D \backslash \bar{D}_{0}}\left|\operatorname{curl} \operatorname{curl} \mathbf{u}_{j}-k_{2}^{2} \mathbf{u}_{j}\right|^{2} d x-k_{2} \int_{D}\left|\operatorname{curl} \mathbf{u}_{j}\right|^{2} d x+k_{2}^{4} \int_{D}\left|\mathbf{u}_{j}\right|^{2} d x=0 .
$$


Define $\mathcal{V}:=\operatorname{Vect}\left\{\mathbf{u}_{j}, 1 \leq j \leq M(r)\right\}$ a $M(r)$-dimensional subspace of $V_{0}\left(D, D_{0}, k\right)$. Let $\mathbf{u} \in \mathcal{V}, \mathbf{u}=\sum_{j=1}^{M(r)} \alpha_{j} \mathbf{u}_{j}$. Since $u_{j}$ and $u_{m}$ have disjoint supports if $j \neq m$, we get

$$
\begin{aligned}
\left(\left(A_{k_{2}}+B_{k_{2}}\right) \mathbf{u}, \mathbf{u}\right)= & \sum_{j=1}^{M(r)} \sum_{m=1}^{M(r)} \alpha_{j} \bar{\alpha}_{m}\left(\left(A_{k_{2}}+B_{k_{2}}\right) \mathbf{u}_{j}, \mathbf{u}_{m}\right) \\
= & \sum_{j=1}^{M(r)}\left|\alpha_{j}\right|^{2}\left(\left(A_{k_{2}}+B_{k_{2}}\right) \mathbf{u}_{j}, \mathbf{u}_{j}\right) \\
\leq & \sum_{j=1}^{M(r)}\left|\alpha_{j}\right|^{2}\left(\frac{1}{N_{*}-1} \int_{D \backslash \bar{D}_{0}}\left|\operatorname{curl} \operatorname{curl} \mathbf{u}_{j}-k_{2}^{2} \mathbf{u}_{j}\right|^{2} d x\right. \\
& \left.-k_{2}^{2} \int_{D}\left|\operatorname{curl} \mathbf{u}_{j}\right|^{2} d x+k_{2}^{4} \int_{D}\left|\mathbf{u}_{j}\right|^{2} d x\right) \\
\leq & 0 .
\end{aligned}
$$

From Theorem 2.7 we deduce that there exist $M(r)$ transmission eigenvalues in $\left[\frac{\lambda_{0}(D)}{N^{*}}, k_{0}\left(r, N_{*}\right)\right]$ counting multiplicity.

The same method shows the result in the case where $(I-N)^{-1}$ is bounded positive definite using $A_{k}^{\prime}$ instead of $A_{k}$.

By letting $r \rightarrow 0$ in the previous theorem we have the following corollary.

COROLlaRY 2.9. There exist infinitely many transmission eigenvalues having $+\infty$ as the only accumulation point.

We denote by $k_{0}\left(D_{0}, N\right)$ the first transmission eigenvalue for the domain $D$ containing the cavity $D_{0}$ and with index $N$ in $D \backslash \bar{D}_{0}$.

Theorem 2.10. If $D_{0} \subseteq D_{0}^{\prime}$ and $N_{2} \leq N_{1}$ then

1. $k_{0}\left(D_{0}, N_{1}\right) \leq k_{0}\left(D_{0}^{\prime}, N_{2}\right)$ if $\left(N_{i}-I\right)^{-1}$ is bounded positive definite for $i=1,2$,

2. $k_{0}\left(D_{0}, N_{2}\right) \leq k_{0}\left(D_{0}^{\prime}, N_{1}\right)$ if $\left(I-N_{i}\right)^{-1}$ is bounded positive definite for $i=1,2$.

Proof. Consider the case where $\left(N_{1}-I\right)^{-1}$ and $\left(N_{2}-I\right)^{-1}$ are both bounded positive definite. First, Theorem 2.7 for the problem with $N=N_{1}$ with $k_{1}:=\frac{\lambda_{0}(D)}{N_{1}^{*}}$ and $k_{2}:=k_{0}\left(D_{0}, N_{2}\right)$ shows that $k_{0}\left(D_{0}, N_{1}\right) \leq k_{0}\left(D_{0}, N_{2}\right)$. We can show in the same way that when $\left(I-N_{1}\right)^{-1}$ and $\left(I-N_{1}\right)^{-1}$ are bounded positive definite, $k_{0}\left(D_{0}, N_{2}\right) \leq$ $k_{0}\left(D_{0}, N_{1}\right)$.

It only remains to show that for all $N$ we have that $k_{0}\left(D_{0}, N\right) \leq k_{0}\left(D_{0}^{\prime}, N\right)$. The proof is similar to the proof of Theorem 2.8. We consider the interior transmission problem for a domain $D$ containing a void $D_{0}$. First, from Theorem 2.3, we know that $A_{k_{1}}+B_{k_{1}}$ is positive for $k_{1}=\frac{\lambda_{0}(D)}{N}$. We define $k_{2}:=k_{0}\left(D_{0}^{\prime}, N\right)$ and let $\mathbf{v} \in$ $V_{0}\left(D, D_{0}^{\prime}, k_{2}\right)$ be an eigenvector corresponding to the eigenvalue $k_{2}$. Since curl curl $\mathbf{v}-$ 
$k_{2}^{2} \mathbf{v}=0$ in $D_{0}^{\prime}$,

$$
\begin{aligned}
\left(A_{k_{2}} \mathbf{v}+B_{k_{2}} \mathbf{v}, \mathbf{v}\right)= & \int_{D \backslash \bar{D}_{0}}(N-1)^{-1}\left(\operatorname{curl} \operatorname{curl} \mathbf{v}-k_{2}^{2} \mathbf{v}\right) \cdot\left(\overline{\operatorname{curl} \operatorname{curl} \mathbf{v}-k_{2}^{2} \mathbf{v}}\right) d x \\
& -k_{2}^{2} \int_{D}|\operatorname{curl} \mathbf{v}|^{2} d x+k_{2}^{4} \int_{D}|\mathbf{v}|^{2} d x \\
= & \int_{D \backslash \bar{D}_{0}^{\prime}}(N-1)^{-1}\left(\operatorname{curl} \operatorname{curl} \mathbf{v}-k_{2}^{2} \mathbf{v}\right) \cdot\left(\overline{\operatorname{curl} \operatorname{curl} \mathbf{v}-k_{2}^{2} \mathbf{v}}\right) d x \\
& -k_{2}^{2} \int_{D}|\operatorname{curl} \mathbf{v}|^{2} d x+k_{2}^{4} \int_{D}|\mathbf{v}|^{2} d x \\
= & 0
\end{aligned}
$$

We deduce that there exists an eigenvalue in $\left[\frac{\lambda_{0}(D)}{N}, k_{0}\left(D_{0}^{\prime}, N\right)\right]$ and consequently for all $N$, we have $k_{0}\left(D_{0}, N\right) \leq k_{0}\left(D_{0}^{\prime}, N\right)$.

3. Appendix. We assume that $D_{0}$ is of class $\mathcal{C}^{2}$. The next theorem shows how we can construct a solution in $L^{2}\left(D_{0}\right)$ to

$$
\begin{cases}\operatorname{curl} \operatorname{curl} \mathbf{E}-k^{2} \mathbf{E}=0 & \text { in } D_{0} \\ \mathbf{E} \times \nu=\alpha \times \nu & \text { on } \partial D_{0} .\end{cases}
$$

with $\alpha \in T H^{-1 / 2}\left(\partial D_{0}\right)$ from a solution in $H\left(\operatorname{curl}, D_{0}\right)$.

THEOREM 3.1. We assume that $k^{2}$ is not an eigenvalue for curlcurl with the boundary condition $\mathbf{E} \times \nu=0$. For every $\alpha \in T H^{-1 / 2}\left(\partial D_{0}\right)$, there exists a solution $\mathbf{E} \in L^{2}\left(D_{0}\right)^{3}$ to

$$
\begin{cases}\operatorname{curl} \operatorname{curl} \mathbf{E}-k^{2} \mathbf{E}=0 & \text { in } D_{0} \\ \mathbf{E} \times \nu=\alpha \times \nu & \text { on } \partial D_{0} .\end{cases}
$$

Proof. We will use the following properties of Sobolev spaces for $D_{0}$ of class $\mathcal{C}^{k+1}$ (see $[9])$ :

$H^{k+1}\left(D_{0}\right)^{3}=\left\{\mathbf{u} \in L^{2}\left(D_{0}\right)^{3}, \operatorname{curl} \mathbf{u} \in H^{k}\left(D_{0}\right)^{3}, \operatorname{div} \mathbf{u} \in H^{k}\left(D_{0}\right), \mathbf{u} \times \nu \in H^{k+1 / 2}\left(\partial D_{0}\right)^{3}\right\}$

or

$H^{k+1}\left(D_{0}\right)^{3}=\left\{\mathbf{u} \in L^{2}\left(D_{0}\right)^{3}, \operatorname{curl} \mathbf{u} \in H^{k}\left(D_{0}\right)^{3}, \operatorname{div} \mathbf{u} \in H^{k}\left(D_{0}\right), \mathbf{u} \cdot \nu \in H^{k+1 / 2}\left(\partial D_{0}\right)^{3}\right\}$.

Let $\alpha \in T H^{1 / 2}\left(\partial D_{0}\right)$ and $\mathbf{E} \in H\left(\operatorname{curl}, D_{0}\right)$ satisfy

$$
\begin{cases}\operatorname{curl} \operatorname{curl} \mathbf{E}-k^{2} \mathbf{E}=0 & \text { in } D_{0} \\ \mathbf{E} \times \nu=\alpha \times \nu & \text { on } \partial D_{0} .\end{cases}
$$

Since $\operatorname{div} \mathbf{E}=0$ and $\mathbf{E} \times \nu \in T H^{1 / 2}\left(\partial D_{0}\right)$, we have that $\mathbf{E}$ is in $H^{1}\left(D_{0}\right)^{3}$. Now let $\mathbf{F} \in H\left(\operatorname{curl}, D_{0}\right)$ be a solution to

$$
\begin{cases}\operatorname{curl} \operatorname{curl} \mathbf{F}-k^{2} \mathbf{F}=\mathbf{E} & \text { in } D_{0} \\ \mathbf{F} \times \nu=0 & \text { on } \partial D_{0} .\end{cases}
$$

We show that $\mathbf{F} \in H^{2}\left(D_{0}\right)^{3}:$ since $\mathbf{F} \in H\left(\operatorname{curl}, D_{0}\right), \operatorname{div} \mathbf{F}=0$ and $\mathbf{F} \times \nu=0$ we have that $\mathbf{F}$ is in $L^{2}\left(D_{0}\right)^{3}, \operatorname{div} \mathbf{F}$ in $H^{1}\left(D_{0}\right)$ and $\mathbf{F} \times \nu$ in $H^{3 / 2}\left(\partial D_{0}\right)^{3}$. Then it only 
remains to show that $\tilde{\mathbf{F}}:=\operatorname{curl} \mathbf{F} \in H^{1}\left(D_{0}\right)^{3}$. The fact that $\mathbf{F} \in H\left(\operatorname{curl}, D_{0}\right)$ implies that $\tilde{\mathbf{F}} \in L^{2}\left(D_{0}\right)^{3}$. The equalities

$$
\operatorname{div} \tilde{\mathbf{F}}=0
$$

and

$$
\operatorname{curl} \tilde{\mathbf{F}}=\mathbf{E}+k^{2} \mathbf{F}
$$

show that $\operatorname{div} \tilde{\mathbf{F}} \in L^{2}\left(D_{0}\right)$ and $\operatorname{curl} \tilde{\mathbf{F}} \in L^{2}\left(D_{0}\right)$. Moreover,

$$
\tilde{\mathbf{F}} \cdot \nu=\operatorname{curl} \mathbf{F} \cdot \nu=\operatorname{div} \partial D_{0}(\mathbf{F} \times \nu)=0
$$

where $\operatorname{div}{ }_{\partial D_{0}}$ denotes the surface divergence operator, implies also that $\tilde{\mathbf{F}} \cdot \nu \in$ $H^{1 / 2}\left(\partial D_{0}\right)$. Then $\tilde{\mathbf{F}} \in H^{1}\left(D_{0}\right)^{3}$ and

$$
\|\tilde{\mathbf{F}}\|_{H^{1}\left(D_{0}\right)} \leq C\left(\|\tilde{\mathbf{F}}\|_{L^{2}\left(D_{0}\right)}^{2}+\|\operatorname{curl} \tilde{\mathbf{F}}\|_{L^{2}\left(D_{0}\right)}^{2}\right)^{1 / 2} \leq C\left(\|\mathbf{F}\|_{H\left(\operatorname{curl}, D_{0}\right)}+\|\mathbf{E}\|_{L^{2}\left(D_{0}\right)}\right) .
$$

We deduce that $\mathbf{F} \in H^{2}\left(D_{0}\right)^{3}$ and

$$
\|\mathbf{F}\|_{H^{2}\left(D_{0}\right)} \leq C\left(\|\mathbf{F}\|_{H\left(\operatorname{curl}, D_{0}\right)}+\|\left.\mathbf{E}\right|_{L^{2}\left(D_{0}\right)}\right) \leq C\|\mathbf{E}\|_{L^{2}\left(D_{0}\right)} .
$$

From (3.1) and using the Stokes formula one easily obtains

$$
\begin{aligned}
\|\mathbf{E}\|_{D_{0}}^{2} & =\left|\int_{\partial D_{0}} \alpha \cdot(\nu \times \operatorname{curl} \mathbf{F})\right| \\
& \leq\|\alpha\|_{H^{-1 / 2}\left(\partial D_{0}\right)}\|\nu \times \operatorname{curl} \mathbf{F}\|_{H^{1 / 2}\left(\partial D_{0}\right)} \\
& \leq C\|\alpha\|_{H^{-1 / 2}\left(\partial D_{0}\right)}\|\mathbf{E}\|_{D_{0}}
\end{aligned}
$$

and therefore the solution operator $\alpha \longmapsto \mathbf{E}$ is continuous from $T H^{-1 / 2}\left(\partial D_{0}\right)$ into $L^{2}\left(D_{0}\right)$. Similar arguments also show that if $k^{2}$ is not an eigenvalue with the boundary condition curl $\mathbf{E} \times \nu=0$ then the solution operator $\operatorname{curl} \alpha \longmapsto \mathbf{E}$ is continuous from $T H^{-3 / 2}\left(\partial D_{0}\right)$ into $L^{2}\left(D_{0}\right)$.

\section{REFERENCES}

[1] F. Cakoni and D. Colton. Qualitative methods in inverse scattering theory. Interaction of Mechanics and Mathematics. Springer-Verlag, Berlin, 2006. An introduction.

[2] F. Cakoni, D. Colton, and H. Haddar. The computation of lower bounds for the norm of the index of refraction in an anisotropic media. J. Integral Equations and Applications, 21(2):203-227, 2009.

[3] F. Cakoni, D. Colton, and H. Haddar. The interior transmission problem for regions with cavities. SIAM J. Math. Anal., 42(1):145-162, 2010.

[4] F. Cakoni, D. Colton, and P. Monk. On the use of transmission eigenvalues to estimate the index of refraction from far field data. Inverse Problems, 23:507-522, 2007.

[5] F. Cakoni, D. Gintides, and H. Haddar. The existence of an infinite discrete set of transmission eigenvalues. SIAM J. Math .Anal., 42:237-255, 2010.

[6] F. Cakoni and H. Haddar. On the existence of transmission eigenvalues in an inhomogeneous medium. Applicable Analysis, 88(4):475-493, 2008.

[7] D. Colton and R. Kress. Inverse acoustic and eletromagnetic scattering theory. Springer, New York, 2nd edition, 1998.

[8] D. Colton, L.Päivärinta, and J. Sylvester. The interior transmission problem. Inverse Problems and Imaging, 1:13-28, 2007.

[9] R. Dautray and J.-L. Lions. Mathematical Analysis and Numerical Methods for Science and Technology, volume 3: Spectral Theory and Applications. Springer-Verlag, Berlin, 1990. 
[10] H. Haddar. The interior transmission problem for anisotropic maxwell's equations and its applications to the inverse problem. Math. Methods Applied Sciences, 27:2111-2129, 2004.

[11] A. Kirsch and N. Grinberg. The factorization method for inverse problems, volume 36 of Oxford Lecture Series in Mathematics and its Applications. Oxford University Press, Oxford, 2008.

[12] L.Päivärinta and J. Sylvester. Transmission eigenvalues. SIAM J. Math. Anal, 40:738-753, 2008.

[13] N. Weck. Approximation by maxwell-herglotz-fields. Math. Methods Applied Sciences, 27:603$621,2004$. 